\title{
IMPLANTATION OF FOLDABLE INTRAOCULAR LENSES IN THE PRESENCE OF ANTERIOR CAPSULAR TEARS
}

\author{
P. M. HAIGH, I. C. LLOYD and M. J. LAVIN \\ Manchester
}

\begin{abstract}
SUMMARY
A consecutive series of patients who had undergone small-incision phacoemulsification cataract surgery with foldable lens implantation were reviewed. Seven patients had sustained anterior capsular tears during surgery. In all cases a three-piece foldable intraocular lens (IOL) was successfully inserted by careful positioning of the haptics. In all 6 patients available for long-term assessment the IOL remained stable and well centred, the tears having been sealed by fibrous tissue. We show that, if logical measures are taken to limit tear extension, the lack of an intact capsulorhexis need not result in conversion to extracapsular cataract extraction.
\end{abstract}

Lens implantation through a small incision has depended on concomitant advances in intraocular lens (IOL) design. Foldable IOLs allow a largediameter optic to be implanted through such an incision. However, implantation of a foldable IOL is recommended only if there is an intact continuous circular capsulorhexis $(\mathrm{CCC})^{1}$ for the following reasons; firstly, the capsulotomy must be strong enough to resist the stretching forces induced as the IOL unfolds within the eye and, secondly, it has been suggested that an intact capsulotomy is necessary to ensure long-term, stable IOL fixation. ${ }^{2}$

This study describes the late post-operative findings in a series of eyes in which a foldable IOL had been implanted despite the presence of a radial anterior capsular tear.

\section{PATIENTS AND METHODS}

We reviewed the case notes of a consecutive series of 34 patients who had undergone small-incision phacoemulsification cataract surgery with foldable IOL implantation between March 1992 and June

Correspondence to: Mr P. M. Haigh, FRCOphth, Manchester Royal Eye Hospital, Oxford Road, Manchester M13 9WH, UK.
1993, and identified 7 patients in whom a foldable lens had been implanted in the presence of a radial anterior capsular tear. The position of the tear and the stage at which it had occurred during the operation were documented. All patients had been operated upon by the same surgeon (M.J.L.) and all had undergone a surgical technique which briefly comprised: a $4 \mathrm{~mm}$ scleral tunnel incision, CCC, hydrodissection, phacoemulsification, automated irrigation/aspiration of cortex and foldable IOL implantation. The IOL implanted in all cases was an Iovision three-piece posterior chamber lens consisting of a $6 \mathrm{~mm}$ diameter silicone optic with blue polypropylene haptics.

When a radial tear had occurred in the edge of the CCC phacoemulsification was performed with extreme care. To prevent extension of the tear into the posterior capsule, the technique was altered as follows: (1) Hydrodissection of the nucleus was performed very slowly with a small volume of fluid so that the nucleus was just delineated, but was not fully mobile. (2) Great care was taken to maintain a deep anterior chamber and prevent sudden shallowing which would produce a pressure differential between the posterior and anterior segments. To minimise fluctuations in the depth of the anterior chamber, a low irrigation flow rate and a low aspiration setting were used. (3) Phacoemulsification sculpting was performed in a direction as far away as possible from the position of the radial tear. With a superior scleral incision and a superior capsular tear the risk of tear extension is least because the direction of sculpting is at approximately $180^{\circ}$ to the tear; the risk is greatest when the tear occurs inferiorly and in these cases the internal opening of the scleral tunnel was enlarged laterally to allow sculpting in a direction away from the tear. (4) If a nuclear cracking technique was used, this was performed very gently and such that the split was produced at $90^{\circ}$ to the direction of the tear.

Eye (1995) 9, 442-445 C 1995 Royal College of Ophthalmologists 
(5) Cortex removal was performed using a low irrigation flow rate and a low aspiration setting.

To successfully implant a foldable three-piece IOL in the presence of a radial tear, a modification of the usual technique was employed. First the IOL was unfolded in the anterior chamber or pupillary plane depending on the depth of the anterior chamber. This was carried out under the protection of a viscoelastic, using a two-handed approach with a second instrument introduced through a side port. The inferior haptic was then carefully guided into the capsular bag at a position away from the tear. The other haptic was then inserted in the bag, if necessary by retracting the edge of the capsulorhexis. Gentle dialling was used to align the IOL correctly in relation to the tear, as long as a haptic was not lying directly under the tear. The IOL had correct alignment when both haptics were in a position away from the capsular tear.

The patients were recalled for examination and the success of IOL implantation was assessed by recording best corrected visual acuity, by measuring IOL decentration and by determining whether the IOL retained capsular bag fixation. To measure IOL decentration, we used a simple method similar to that described by Caballero and co-workers. ${ }^{3}$ Eyes were fully dilated using one drop each of cyclopentolate $1 \%$ and phenylephrine $2.5 \%$, and were then photographed axially to show the implant against the red reflex. The photographic slides were projected onto white paper upon which was drawn a $12 \mathrm{~cm}$ diameter circle. The magnification was then adjusted until the edge of the IOL corresponded exactly with the template outline and the position of the limbus was drawn onto the paper. A circle of best fit was matched to the sketch of the limbus and the distance between the centre of this circle and the centre of the template was measured in millimetres. After dividing by 20 the IOL decentration in millimetres was obtained.

\section{RESULTS}

In 4 of the 7 patients a radial tear had occurred in the anterior capsule during fashioning of the CCC; the tear was in the inferotemporal quadrant in 3 eyes and in the superonasal quadrant in 1 eye. In 2 patients, radial anterior capsular tears had occurred during phacoemulsification, in one when the instrument tip touched the edge of the CCC, and in the other when a hard nuclear fragment rotated forcibly within the capsular bag splitting the capsulorhexis edge; both tears were located superiorly. In the final patient identified, a radial anterior capsular tear had been produced in the inferonasal quadrant as the IOL unfolded within the eye. In all eyes the IOL was positioned so that the haptics were lying away from the capsular tear. In our series there were no cases with vitreous loss or nuclear dislocation due to a radial tear and none of the tears had extended beyond the equator of the lens to involve the posterior capsule.

Six of the 7 patients attended for review a mean of 14.7 months post-operatively (Table I); the other patient had died. The late post-operative appearances of a tear in the inferotemporal quadrant (Fig. 1a) and in the superonasal quadrant (Fig. 1b) are shown. Capsular fixation of the IOL had been maintained in all 6 eyes and, most interestingly, all the anterior capsular tears had become bridged by fibrous tissue, an example of which is shown in Fig. 1b. Best corrected visual acuity, decentration and astigmatism data are summarised in Table I. None of the 6 eyes had discoloration of the IOL, pigment dispersion, inflammatory deposits, posterior synechiae or lens capture. No patient required YAG laser capsulotomy during this period of follow-up and none had experienced a sudden change in refraction.

\section{DISCUSSION}

When a radial tear occurs in the anterior capsule the surgeon has two main objectives: firstly, to prevent extension of the tear up to or beyond the equator and, secondly, to position the IOL in such a way that secure capsular fixation will result, ensuring longterm stability and centration. Irrespective of the initiating insult, all anterior radial tears tend to behave in a similar manner. When an interruption or loss of integrity appears in the smooth edge of the $\mathrm{CCC}$ it extends rapidly towards the equator (Fig. 2a), particularly if the pressure differential between the posterior and anterior segments is relatively high at that time. ${ }^{4}$ Strong zonular forces in the region of the

Table I. Best corrected visual acuity, decentration and astigmatism in 6 eyes

\begin{tabular}{ccccc}
\hline Case no. & BCVA & Decentration (mm) & Astigmatism (dioptres) \\
\hline 1 & $6 / 5$ & 0.45 & 0.75 & Follow-up (months) \\
2 & $6 / 6$ & 0.25 & 1.25 & 0.50 \\
3 & $6 / 9$ & 0.00 & 11 & 2.00 \\
4 & $6 / 9$ & 0.20 & 2.00 & 22 \\
5 & $6 / 9$ & 0.35 & 0.50 & Mean = 1.08 \\
$6^{\text {a }}$ & $6 / 60$ & Mean = 0.29 & Mean = 14.7 \\
\hline
\end{tabular}

BCVA, best corrected visual acuity.

${ }^{a}$ Coexistent diabetic maculopathy. 


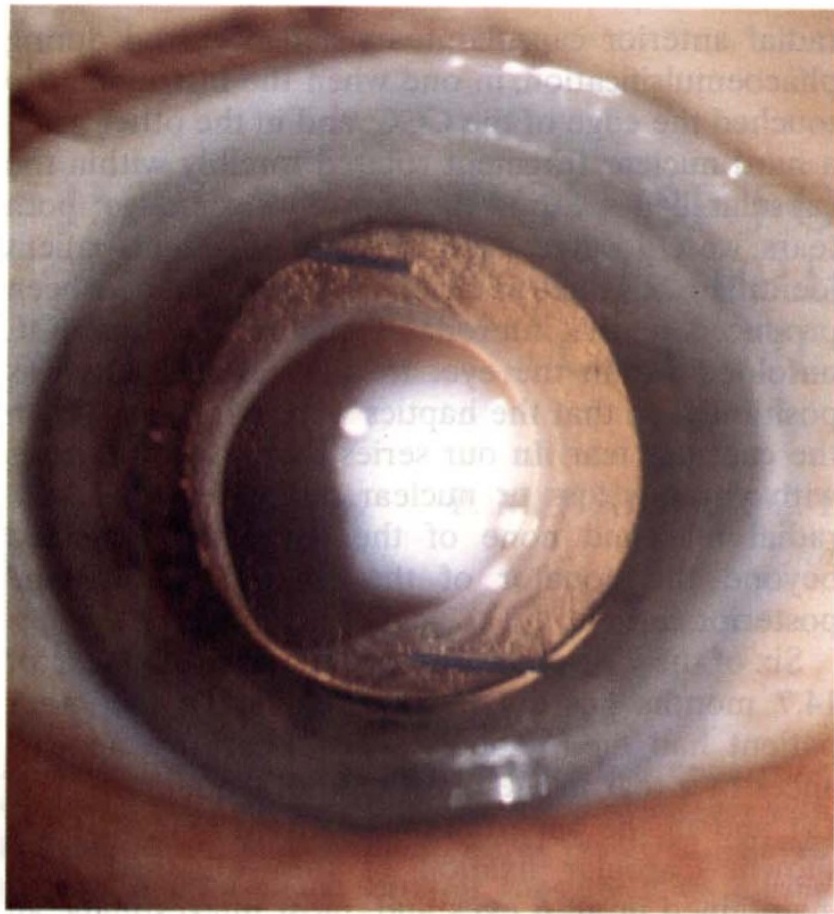

(a)

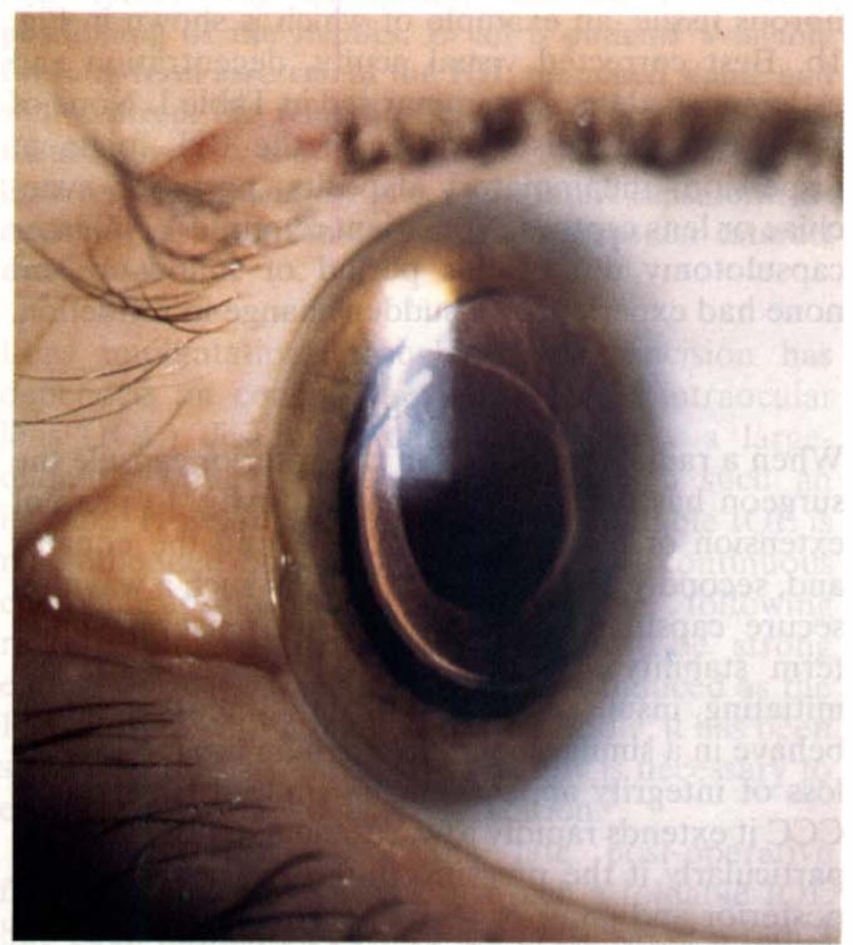

(b)

Fig. 1. Late post-operative appearances of anterior capsular tears. (a) Tear in the inferotemporal quadrant. (b) Superonasal tear bridged by fibrous tissue.

equator then resist further extension of the tear into the posterior capsule (Fig. 2b). However, it has been shown that, if additional force is exerted, the bridging zonular fibres will break and the tear will extend. ${ }^{5}$

The aim of the surgeon, therefore, is to keep to a minimum any tangentially applied forces on the flaps

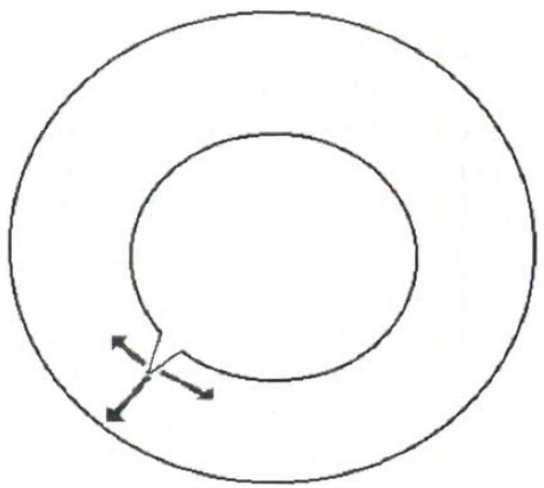

(a)

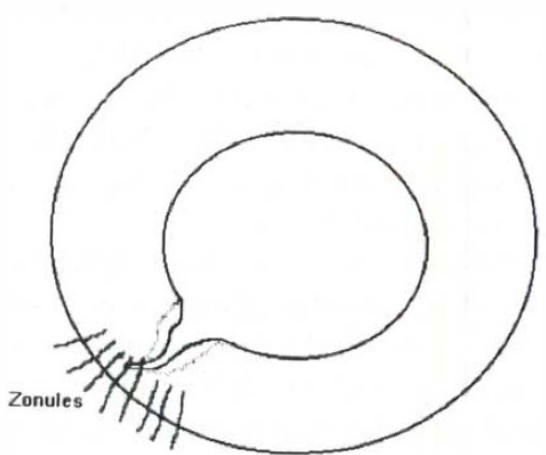

(b)

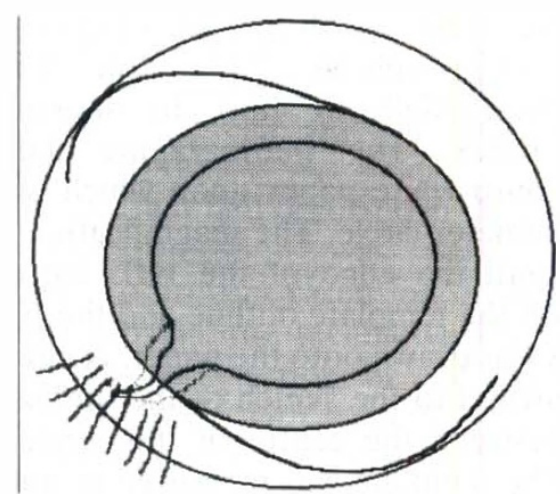

(c)

Fig. 2. (a) Vector forces acting on an anterior radial tear. (b) Bridging zonular fibres resisting extension of the radial tear. (c) IOL haptics orientated away from the radial tear. The static elastic forces exerted by the polypropylene haptics are too weak to disrupt the bridging zonular fibres. (After Assia et al..$^{5}$ )

of the tear in order to preserve zonular integrity. When the capsulorhexis is not intact phacoemulsification must be performed with extreme care, in order to prevent tear extension resulting in vitreous loss or posterior nuclear dislocation. In our series, using the technique described, phacoemulsification was performed safely without complications, but we emphasise that there should be a low threshold for conversion to extracapsular extraction if zonular disruption is suspected.

The static elastic forces exerted by the IOL haptics when positioned away from the radial tear appear to 
be insufficient to disrupt the zonule and hence do not cause the tear to extend posteriorly (Fig. 2c). The magnitude of the static forces generated by different types of haptic have been measured in vitro ${ }^{6}$ but the force required to disrupt the bridging zonular fibres to allow a radial tear to extend posteriorly has not been quantified. We postulate that in our series the forces generated by the IOL haptics were smaller than the forces needed to break the zonular fibres, and the edges of the tears remained sufficiently apposed to allow bridging by fibrous tissue. This phenomenon of sealing of an anterior tear probably occurs as a manifestation of capsular bag contraction. ${ }^{7}$ Sealed tears have been observed previously in a study on post-mortem eyes ${ }^{8}$ but, to our knowledge, have not been demonstrated before in vivo.

It is now well recognised that a radial tear can allow one of the haptics to dislocate from the capsular bag post-operatively, resulting in bag-sulcus IOL fixation. ${ }^{9-11}$ This mode of fixation may generate considerable decentration as capsular fibrosis and shrinkage occur. By placing the haptics away from the radial tear capsular bag fixation is much more likely to be maintained, ensuring consistent centration. ${ }^{11}$ Furthermore, when fibrous bridging of the radial tear occurs the integrity of the capsular opening is reconstituted, effectively preventing any late extrusion of a haptic from the capsular bag. In this respect anterior capsular fibrosis and sealing of radial tears are important in contributing to the maintenance of stable capsular IOL fixation.

It has been stated that, for soft IOLs, stable fixation and good centration are possible only in the presence of an intact capsulorhexis. ${ }^{2,12,13}$ However, all 6 of our patients had a good outcome. All IOLs were stable and had retained capsular fixation at the late follow-up visit. Five of the patients had good visual acuity, the exception being a patient with coexistent diabetic maculopathy. The IOLs were well centred with a mean decentration of only $0.29 \mathrm{~mm}$, and this value concurs with that found by other workers for polymethylmethacrylate IOLs implanted 'within the bag' in the presence of a single anterior capsular tear. 9,10

We conclude that a three-piece foldable IOL can be successfully implanted in the capsular bag despite the presence of a radial anterior capsular tear. We feel it is safe to proceed with foldable IOL implantation, and thus avoid conversion to extracapsular cataract extraction, so long as the zonule remains intact in the region of the tear.

Key words: Capsular tears, Foldable intraocular lenses, Phacoemulsification.

\section{REFERENCES}

1. Product information sheet. Iovision foldable lens, Iovision Inc., Irvine, CA 92718.

2. Shimizu K, Komatsu M. Physical fixation of soft intraocular lenses. J Cataract Refract Surg 1989; 15:580-3.

3. Caballero A, Losada M, Lopez MC. A simple method for measuring intraocular lens decentration in vivo. Eur J Implant Refract Surg 1993;5:137-8.

4. Luck J, Coster DJ. Capsular tearing and peripheral tear extension in capsulorhexis: a vector analysis. AsiaPacific J Ophthalmol 1994;6:12-5.

5. Assia EI, Apple DJ, Tsai JC, Morgan RC. Mechanism of radial tear formation and extension after anterior capsulectomy. Ophthalmology 1991;98:432-7.

6. Guthoff R, Abramo F, Draeger J, Chumbley L. Measurement of elastic resisting forces of intraocular haptic loops of varying geometrical designs and material composition. J Cataract Refract Surg 1990; 16:551-8.

7. Davison JA. Capsule contraction syndrome. J Cataract Refract Surg 1993;19:582-9.

8. Wasserman D, Apple DJ, Castaneda VE, Tsai JC, Morgan RC, Assia EI. Anterior capsular tears and loop fixation of posterior chamber intraocular lenses. Ophthalmology 1991;98:425-31.

9. Caballero A, Lopez MC, Losada M. Behaviour of intraocular lenses implanted after capsulorhexis and envelope capsulotomy. I. Postoperative IOL displacement. Eur J Implant Refract Surg 1993;5:88-94.

10. Assia EI, Legler UFC, Merrill C, Hicklin JC, Castaneda VE, Hoggart JP, et al. Clinicopathologic study of the effect of radial tears and loop fixation on intraocular lens decentration. Ophthalmology 1993; 100:153-8.

11. Davison JA. Analysis of capsular bag defects and intraocular lens positions for consistent centration. J Cataract Refract Surg 1986;12:124-9.

12. Shepherd JR. Continuous-tear capsulotomy and insertion of a silicone bag lens. J Cataract Refract Surg 1989;15:335-9.

13. Menapace R, Amon M, Papapanos P, Radax U. Evaluation of the first 100 consecutive PhacoFlex silicone lenses implanted in the bag through a selfsealing tunnel incision using the Prodigy inserter. J Cataract Refract Surg 1994;20:299-309. 